PAPER

\title{
Vagus nerve stimulation: predictors of seizure freedom
}

\author{
J Janszky, M Hoppe, F Behne, I Tuxhorn, H W Pannek, A Ebner
}

J Neurol Neurosurg Psychiatry 2005;76:384-389. doi: 10.1136/jnnp.2004.037085

\begin{abstract}
Objectives: To identify predictive factors for the seizure-free outcome of vagus nerve stimulation (VNS). Methods: All 47 patients who had undergone VNS implantation at one centre and had at least one year of follow up were studied. They underwent complete presurgical evaluation including detailed clinical history, magnetic resonance imaging, and long term video-EEG with ictal and interictal recordings. After implantation, adjustment of stimulation parameters and concomitant antiepileptic drugs were at the discretion of the treating physician. Results: Mean (SD) age of the patients was 22.7 (11.6) years (range 7 to 53 ). Six patients (13\%) became seizure-free after the VNS implantation. Only two variables showed a significant association with the seizure-free outcome: absence of bilateral interictal epileptiform discharges (IED) and presence of malformation of cortical development (MCD). Epilepsy duration showed a non-significant trend towards a negative association with outcome. By logistic regression analysis, only absence of bilateral IED correlated independently with successful VNS treatment $(p<0.01$, odds ratio $=29.2(95 \%$ confidence interval, 2.4 to 353)). Bilateral IED (independent or bilateral synchronous) was found in one of six seizure-free patients and in 33 of 41 non-seizure-free patients. When bilateral IED were absent, the sensitivity for seizure-free outcome was 0.83 (0.44 to 0.97$)$, and the specificity was 0.80 (0.66 to 0.90$)$. Conclusions: Bilateral IED was independently associated with the outcome of VNS. These results are preliminary because they were based on a small patient population. They may facilitate prospective VNS studies enrolling larger numbers of patients to confirm the results.
\end{abstract}

See end of article for authors' affiliations

Correspondence to: Dr Alois Ebner, EpilepsieZentrum Bethel, Mara Krankenhaus, Maraweg 21, Bielefeld 33617, Germany; ae@mara.de

Received 30 January 2004 In revised form 26 June 2004 Accepted 11 July 2004

V gus nerve stimulation (VNS) has been shown to be effective treatment for drug resistant epilepsy in two randomised, double blind studies. ${ }^{2}{ }^{2}$ During recent years VNS has become an accepted method of treating patients with refractory epilepsy who are not candidates for epilepsy surgery or in whom surgery resulted in no benefit. From the experience of centres using VNS, one third of patients have a reduction in seizure frequency of at least $50 \%$, another third have a worthwhile seizure reduction, while in the remaining third there is little or no effect. ${ }^{3}$ There is only limited information on patients who become seizure-free. ${ }^{3}$ The rate of seizure freedom shows a large variation across the studies which have provided any information at all about it, and ranged from $0 \%$ to $24 \% .^{4-13}$

Despite increasing amounts of clinical data, it is still not possible to predict which patients will profit from VNS. ${ }^{14}$ Determining prognostic factors is important in predicting seizure outcome of VNS and in counselling patients. Moreover, because of the invasive nature of the procedure, the possible hazards of chronic implantation, and the relatively high cost of the treatment, factors indicating a poor prognosis could favour other treatment options including the newer antiepileptic drugs, palliative epilepsy surgery, non-surgical non-pharmacological treatment, or a ketogenic diet. The identification of prognostic factors could also improve our general understanding of the physiological mechanisms of the effect of VNS on seizures.

In two studies focusing on epilepsy duration it was found that patients with a longer duration of epilepsy had a significantly smaller chance of being seizure-free on VNS treatment than those with epilepsy duration of less than six years. ${ }^{7}{ }^{15}$ In contrast, in a study involving a search of the VNS outcome treatment registry-a database created by participating prescribing physicians who voluntarily submit baseline information on patient treatment to a centralised data storage site $^{10}$-it was found that VNS responsiveness was associated with older age, longer epilepsy duration, and epilepsy syndromes other than Lennox-Gastaut syndrome. ${ }^{10}$ Some other studies have unsuccessfully tried to identify prognostic factors for a seizure reduction of more than 50\% by VNS treatment. ${ }^{12}{ }^{13}$ Moreover, in all studies analysing the efficacy of VNS, a 50-75\% reduction or percentage change in seizure frequency rather than seizure freedom has been the dominant end point. ${ }^{12}{ }^{12}$ 16-18 Conversely, health related quality of life (HQOL) is primarily related to seizure freedom and not to seizure reduction. In a study evaluating the relation of seizure improvement to HQOL after introducing a new antiepileptic drug, HQOL improvement was consistently evident only among patients achieving seizure freedom and not in those who had a $50-75 \%$ or $75-99 \%$ seizure reduction. ${ }^{19}$ Although epilepsy patients experience significant impairment in their HQOL, seizure-free patients can have HQOL levels comparable to those of the general population, which supports striving for seizure freedom as an epilepsy care goal..$^{20}$

Most studies investigating the outcome of VNS do not require complete presurgical evaluation. A recent study reported that four of 11 patients who were referred for VNS because of "drug resistant epilepsy" without presurgical evaluation actually had no epilepsy at all or had been good candidates for epilepsy surgery, which was obvious only after a presurgical evaluation that included video-EEG monitoring. ${ }^{21}$ Moreover, more detailed data provided by presurgical evaluation may lead to greater insight into the predictors of VNS success. Our study was aimed at identifying predictive factors for seizure-free outcome after VNS in 47 patients who had undergone extensive presurgical evaluation including magnetic resonance imaging (MRI) and long term videoEEG.

Abbreviations: $H Q O L$, health related quality of life; IED, interictal epileptiform discharge; MCD, malformation of cortical development; VNS, vagus nerve stimulation 


\begin{tabular}{|c|c|c|c|c|}
\hline & $\begin{array}{l}\text { All patients } \\
(n=47)\end{array}$ & $\begin{array}{l}\text { Seizure-free } \\
(n=6)\end{array}$ & $\begin{array}{l}\text { Non-seizure-free } \\
(n=41)\end{array}$ & p Value \\
\hline Age at implantation (years) & $22.7(11.6)(7$ to 53$)$ & $17.5(13.3)$ & $23.5(11.2)$ & 0.16 \\
\hline Age at onset (years) & $4.9(4.6)(0$ to 18$)$ & $6.0(3.9)$ & $4.8(4.7)$ & 0.26 \\
\hline Duration of illness (years) & $17.8(10)(3$ to 44$)$ & $11.5(10.0)$ & $18.7(9.7)$ & 0.07 \\
\hline Women & $25(53 \%)$ & $3(50 \%)$ & $22(54 \%)$ & 1.0 \\
\hline Men & $22(47 \%)$ & $3(50 \%)$ & $19(46 \%)$ & 1.0 \\
\hline Previous epilepsy surgery & $14(30 \%)$ & $1(17 \%)$ & $13(32 \%)$ & 0.65 \\
\hline
\end{tabular}

\section{METHODS}

\section{Patient selection}

For this retrospective study we included all patients in whom VNS implantation had been carried out in our centre between 1 January 2000 and 31 October 2002, and who had at least one year of follow up. Neither our centre nor our patients had participated in previous double blind randomised studies. All patients were referred to our centre for presurgical evaluation because of chronic drug resistant epilepsy and were selected for VNS on the basis of their prognosis for resective brain surgery. We selected only those patients who had undergone a complete presurgical evaluation including a detailed clinical history, MRI, and long term video-EEG with ictal and interictal recordings.

\section{Presurgical evaluation at the epilepsy surgery department of the Bethel epilepsy centre}

As the observed variables were based on presurgical evaluation of epileptic patients, we shall describe our protocol briefly. In patients who were to be considered possible candidates for epilepsy surgery, a detailed neurological history was taken. Resistance to first line antiepileptic drugs was evaluated. A high resolution MRI was carried out in all patients. In those with negative MRI, a second and more detailed MRI investigation was undertaken, focusing more on the region where the epileptogenic lesion was thought to be located, based on the presurgical evaluation. (the technical details of MRI investigation are described below). Patients underwent continuous video-EEG monitoring lasting two to 10 days. In all patients except small children, psychiatric and neuropsychological examination and a social assessment were undertaken. Findings of the presurgical evaluation were discussed at a multidisciplinary case conference, where decisions were made about the possibility and type of brain surgery or VNS, and on the need for further investigations.

\section{Vagus nerve stimulation}

Stimulation of the left vagal nerve was carried out using the NCP system (Cyberonics Inc, Houston, Texas, USA). The system and surgical procedure have been described elsewhere previously. ${ }^{22}$ Stimulation was initiated in the immediate postoperative period, using the following parameters: current $0.25 \mathrm{~mA}$, frequency $30 \mathrm{~Hz}$, pulse width $500 \mu \mathrm{s}$, $30 \mathrm{~s}$ signal on time, 5 min signal off time. Patients in whom a vagus nerve stimulator had been implanted were usually examined every one to three months. At every follow up visit seizure frequency, seizure type, antiepileptic drug regimen change, and any side effects of the treatment were assessed. On these subsequent visits the current was gradually increased by 0.25 to $0.5 \mathrm{~mA}$ per visit until the individual patient's tolerance, seizure freedom, or a maximum of $3.5 \mathrm{~mA}$ were reached. The other device parameters usually remained unchanged except for the signal on and off time. Patients who had no seizure reducing effect 0.5 to 1.5 years after implantation by changing the output current were offered the "rapid cycle mode" which consisted of on/off periods of $7 \mathrm{~s} / 14 \mathrm{~s}$. No restrictions were placed on changes of the concomitant antiepileptic drug. The adjustment of stimulation parameters and concomitant antiepileptic drug were at the discretion of the treating physician. The patients were also provided with a magnet allowing additional stimulation to be commanded by the patient or a caregiver in the case of an aura or seizure. The magnet output current was programmed at $0.25 \mathrm{~mA}$, higher than the automatically delivered stimulation, with a pulse width of $500 \mu$ s for a period of 60 seconds.

\section{Data collection}

We investigated the following groups of variables: general data, seizure semiology, EEG and neuroimaging data, and epilepsy syndrome. General data were obtained from the clinical history and medical records. The list of variables is presented in table 1. The variables of seizure semiology were based on video-EEG recordings, while aura, the presence of secondarily generalised tonic-clonic seizures (SGTCS), number of seizure types, and seizure frequency were obtained from the clinical history (see list of variables in table 2). $E E G$ data (table 3 ) were determined from non-invasive continuous video-EEG monitoring. Preoperative 32-64 channel EEG recordings were used; electrodes were placed according to the 10-10 system, ${ }^{23}$ the number of electrodes and their placement being varied individually according to the suspected epileptogenic region and side. The location and

Table 2 Seizure characteristics

\begin{tabular}{|c|c|c|c|c|}
\hline & $\begin{array}{l}\text { All patients } \\
(n=47)\end{array}$ & $\begin{array}{l}\text { Seizure-free } \\
(n=6)\end{array}$ & $\begin{array}{l}\text { Non-seizure-free } \\
(n=41)\end{array}$ & $p$ Value \\
\hline Presence of auras & $26(55 \%)$ & $3(50 \%)$ & $23(57 \%)$ & 1.0 \\
\hline Complex partial seizures & $25(53 \%)$ & $3(50 \%)$ & $22(54 \%)$ & 1.0 \\
\hline Tonic seizures & $21(45 \%)$ & $1(17 \%)$ & $20(49 \%)$ & 0.20 \\
\hline Clonic seizures & $6(13 \%)$ & $1(17 \%)$ & $5(12 \%)$ & 1.0 \\
\hline Myoclonic seizures & $9(19 \%)$ & $2(33 \%)$ & $7(17 \%)$ & 0.32 \\
\hline Atypical absences & $7(15 \%)$ & $1(17 \%)$ & $6(14 \%)$ & 1.0 \\
\hline Secondary generalization & $20(43 \%)$ & $1(17 \%)$ & $19(46 \%)$ & 0.22 \\
\hline \multicolumn{5}{|l|}{ Seizure frequency } \\
\hline$>1 / d$ & $25(53 \%)$ & $4(67 \%)$ & $21(51 \%)$ & 0.67 \\
\hline One seizure type only & $27(57 \%)$ & $4(67 \%)$ & $23(57 \%)$ & 1.0 \\
\hline
\end{tabular}


Table 3 EEG data

\begin{tabular}{lllll}
\hline & $\begin{array}{l}\text { All patients } \\
(\mathbf{n = 4 7 )}\end{array}$ & $\begin{array}{l}\text { Seizure-free } \\
(\mathbf{n = 6})\end{array}$ & $\begin{array}{l}\text { Non-seizure-free } \\
(\mathbf{n}=\mathbf{4 1 )}\end{array}$ & p Value \\
\hline Non-lateralised seizure pattern & $15(32 \%)$ & $0(0 \%)$ & $15(37 \%)$ & 0.16 \\
Bilateral independent seizure pattern & $8(17 \%)$ & $0(0 \%)$ & $8(20 \%)$ & 0.57 \\
$\quad$ Absence of bilateral IED & $13(28 \%)$ & $5(83 \%)$ & $8(20 \%)$ & 0.004 \\
Bilateral independent IED & $25(53 \%)$ & $1(17 \%)$ & $24(59 \%)$ & 0.08 \\
$\quad$ Generalised/bilateral synchronous IED & $18(38 \%)$ & $1(17 \%)$ & $17(41 \%)$ & 0.38 \\
\hline Values are $\mathrm{n}(\%)$. & & & \\
IED, interictal epileptiform discharges. & & & \\
\hline
\end{tabular}

frequency of interictal epileptiform discharges (IED) were assessed by visual analysis of interictal EEG samples of two minutes duration per hour.

\section{Structural MRI investigations}

MRI scanning was carried out on a $1.5 \mathrm{~T}$ scanner equipped with ultra gradients, a standard head coil, and vacuum cushions to reduce patient movement (Siemens Magnetom Symphony, Erlangen, Germany). The MRI investigation for epilepsy consisted of coronal and axial FLAIR datasets (time of repetition $(\mathrm{TR})=9000 \mathrm{~ms}$, time of echo $(\mathrm{TE})=110 \mathrm{~ms}$, inversion time $(\mathrm{TI})=2500 \mathrm{~ms}$, slice thickness $5 \mathrm{~mm}$, field of view (FOV) $201 \times 230$, matrix $220 \times 256$ ); coronal proton density and $\mathrm{T} 2$ weighted images $(\mathrm{TR}=3075 \mathrm{~ms}, \mathrm{TE}=14 / 85$ ms, slice thickness $5 \mathrm{~mm}$, FOV $201 \times 230$, matrix $210 \times 256$ ); axial $\mathrm{T} 2$ weighted images $(\mathrm{TR}=5401 \mathrm{~ms}, \mathrm{TE}=90 \mathrm{~ms}$, slice thickness $5 \mathrm{~mm}$, FOV $230 \times 230$, matrix $270 \times 512$ ); and a Tl weighted coronal three dimensional sequence (MPRAGE, $\mathrm{TR}=11.1 \mathrm{~ms}$, $\mathrm{TE}=4.3 \mathrm{~ms}$, slice thickness $1.5 \mathrm{~mm}$, FOV $201 \times 230 \mathrm{~mm}$, matrix $224 \times 256$ ). T2 relaxometry or proton MR spectroscopy was undertaken in many patients; however, the results of these investigations were not evaluated in the present study.

\section{Outcome assessment}

Outcome was assessed at regular postoperative visits. We only evaluated the seizure outcome, and psychiatric, social, and neuropsychological outcomes were ignored. For this study we divided the patients into the two categories, seizurefree and non-seizure-free. Patients who had non-disabling auras exclusively were considered to be seizure-free. Seizure freedom was defined if the patient was seizure-free more than three months before the last postoperative visit. If seizures reappeared in an initially seizure-free patient, the outcome was defined as non-seizure-free.

\section{Statistical methods}

For the single predictive variable analyses using categorical variables, $\chi^{2}$, Fisher's exact, and binomial tests were used. The Mann-Whitney U test was employed for comparisons of continuous variables. The non-parametric tests were used because variables were not distributed normally. To identify which variables could predict the outcome independently, we carried out a logistic regression for variables showing a significant effect in single predictive variable analyses. Two tailed error probabilities smaller than $\mathrm{p}<0.05$ were considered significant.

\section{RESULTS}

Three patients were excluded because they had no video-EEG monitoring before VNS implantation and 47 patients met the inclusion criteria ( 25 women and 22 men). Their mean (SD) age was 22.7 ( 11.6 ) years (range 7 to 53 ). Mean age at disease onset was 4.9 (4.6) years (range 0 to 18 ). Fourteen patients had previously had unsuccessful epilepsy surgery. Table 2 presents the seizure characteristics of the patient population. Complex partial seizures were the most common seizure type. Secondary generalisation of seizures was present in $43 \%$. Table 3 shows the EEG data. In $72 \%$ of patients, bilateral independent or bilateral synchronous IED were present. Table 4 shows the MRI data. In 26\% of the patients no structural abnormalities were detected. The most common abnormalities were malformations of cortical development (MCD), occurring in 34\% of patients, while hippocampal sclerosis was present in $11 \%$; perinatal stroke, tuberous sclerosis, and hypothalamic hamartoma occurred in $6 \%$ of the patients. Most patients had extratemporal focal epilepsy (see table 5).

Six of 47 patients (13\%) became seizure-free after the VNS implantation. None of the three patients who were previously excluded owing to lack of video-EEG monitoring became seizure-free. The mean (SD) follow up time was 22 (7) months (range 12 months to 3 years). In the seizure-free patients, the mean follow up time was 27 (5) months; in the non-seizure-free it was 21 (7) months. This difference showed a non-significant trend $(p=0.08)$. In the seizurefree patients the mean current output was 1.5 (0.6) $\mathrm{mA}$ (range 0.5 to 2 ); in non-seizure-free patients it was $1.9(0.6)$ $\mathrm{mA}$ (range 0.75 to 3.5 ), $\mathrm{p}=0.3$. At the time of the evaluation, all the six seizure-free patients had been seizure-free for more than six months (mean 11 months, range 6 to 20). There were no patients who initially became seizure-free (that is, more than three months of seizure freedom) and later experienced a seizure recurrence. The time for achieving seizure freedom ranged from 0 to 21 months (mean 16 months).

In 23 patients, we changed the standard cycle parameters to rapid cycle parameters and three became seizure-free;

Table 4 MRI data

\begin{tabular}{lllll}
\hline & $\begin{array}{l}\text { All patients } \\
(\mathbf{n = 4 7 )}\end{array}$ & $\begin{array}{l}\text { Seizure-free } \\
(\mathbf{n = 6 )}\end{array}$ & $\begin{array}{l}\text { Non-seizure-free } \\
(\mathbf{n = 4 1 )}\end{array}$ & p Value \\
\hline No detectable pathology on MRI & $12(26 \%)$ & $1(17 \%)$ & $11(27 \%)$ & 1.0 \\
Hippocampal sclerosis & $5(11 \%)$ & $1(17 \%)$ & $4(10 \%)$ & 0.51 \\
Malformations of cortical development & $16(34 \%)$ & $5(83 \%)$ & $11(27 \%)$ & 0.013 \\
Perinatal stroke & $3(6 \%)$ & $0(0 \%)$ & $3(7 \%)$ & 1.0 \\
Tuberous sclerosis & $3(6 \%)$ & $0(0 \%)$ & $3(7 \%)$ & 1.0 \\
Hypothalamic hamartoma & $3(6 \%)$ & $0(0 \%)$ & $3(7 \%)$ & 1.0 \\
\hline Values are $\mathrm{n}(\%)$. & & & \\
\hline
\end{tabular}




\begin{tabular}{|lllll}
\hline \multicolumn{7}{l}{ Table 5} & Epilepsy syndrome & & \\
\hline & $\begin{array}{l}\text { All patients } \\
(\mathbf{n}=\mathbf{4 7})\end{array}$ & $\begin{array}{l}\text { Seizure-free } \\
(\mathbf{n}=6)\end{array}$ & $\begin{array}{l}\text { Non-seizure-free } \\
(\mathbf{n}=\mathbf{4 1 )}\end{array}$ & $\mathbf{p}$ Value \\
\hline Temporal lobe epilepsy & $12(26 \%)$ & $2(33 \%)$ & $10(24 \%)$ & 0.63 \\
Extratemporal focal epilepsy & $31(66 \%)$ & $4(67 \%)$ & $27(66 \%)$ & 1.0 \\
Symptomatic generalised epilepsy & $4(9 \%)$ & $0(0 \%)$ & $4(10 \%)$ & 1.0 \\
\hline
\end{tabular}

three of the 24 patients who did not receive rapid cycle stimuli also became seizure-free $(\mathrm{p}=1.0)$.

\section{Single predictive variable analyses}

Only two variables showed a significant association with the seizure-free outcome (see tables 1-5): absence of bilateral IED and presence of MCD. Concerning the absence of bilateral IED, the sensitivity for a seizure-free outcome was 0.83 (95\% confidence interval (CI), 0.44 to 0.97 ); while the specificity was 0.8 (0.66 to 0.9$)$. In the case of MCD, the sensitivity for a seizure-free outcome was 0.83 (0.44 to 0.97 ), while the specificity was 0.73 ( 0.58 to 0.84$)$. The duration of illness showed a non-significant trend towards a negative association with outcome. It is important to note that owing to the relatively small number of patients the sensitivity and specificity data provide only limited information, and further data will be needed to get really reliable estimates.

\section{The possible effect of new antiepileptic drug}

During the follow up, new antiepileptic drugs were added in 11 patients $(23 \%)$-in two of six patients who became seizure-free and in nine of the remaining patients $(p=0.6)$. In both seizure-free patients, the new add-on antiepileptic drug was levetiracetam. In the first case, the 35 year old male patient was initially seizure-free on $0.75 \mathrm{~mA}$ current output during VNS. Two months later the seizures returned and increasing the current output resulted in no further benefit. Levetiracetam was added and the patient then became seizure-free. In the second case, the nine year old girl suffering from intractable temporal lobe epilepsy had a $>50 \%$ seizure reduction during VNS treatment with the standard cycle mode. After introduction of a rapid cycle mode, a slight further improvement was seen. One year later, however, there was no more change in the seizure situation; thus levetiracetam was added as a new antiepileptic drug and this resulted in seizure freedom. Conversely, there were four other patients who did not become seizure-free and who received levetiracetam as a new antiepileptic drug. The possible positive effect of the newly introduced levetiracetam on the seizure-free outcome was not significant $(p=0.16)$.

Concerning those 35 patients in whom MRI abnormalities were visualised, seven had bilateral abnormalities and none became seizure-free, while five of the remaining 28 patients with unilateral pathology became seizure-free (table 4). This difference, however, was not statistically significant $(\mathrm{p}=0.6)$. Of those 28 patients with unilateral pathology on MRI, four of 14 patients with left sided and one of 14 with right sided abnormalities achieved seizure freedom $(p=0.3)$.

\section{Independence of variables and logistic regression analysis}

Stepwise logistic regression was undertaken to investigate the two factors that were found to be significant in single predictive variable analyses. Only the absence of bilateral IED correlated independently with successful VNS treatment ( $p<0.01$, odds ratio 29.2 (95\% CI, 2.4 to 353); the presence of MCD did not.

\section{DISCUSSION}

In our study, the absence of bilateral spike focus and malformation of cortical development on MRI were associated with successful VNS treatment according to the single predictive variable analysis. However, logistic regression analysis showed that only the absence of bilateral interictal epileptiform discharges was independently associated with the outcome of VNS; the presence of cortical malformations was not. Age at epilepsy onset, duration of epilepsy, seizure type, seizure frequency, epilepsy syndrome, or other MRI abnormalities showed no significant association with the outcome of VNS treatment. However, our patient population was relatively small, so some infrequent clinical phenomena might have had no chance of reaching statistical significance. Interestingly, our results are in agreement with epilepsy surgery studies showing that EEG is a reliable tool for assessing outcome prognosis. ${ }^{24-27}$

Some studies have shown that patients with bitemporal epilepsy (such as bitemporal independent seizure onset in one patient) may benefit from VNS treatment; however, no seizure-free patients were reported. ${ }^{28}{ }^{29}$ In our study, none of the patients who had bilateral independent seizures became seizure-free. However, this result was not statistically significant (table 3). Conversely, we found that the absence of bilateral (independent and bilateral synchronous) IED showed an association with a favourable outcome of VNS treatment. Interestingly, absence of bilateral IED is also a good prognostic factor for resective epilepsy surgery according to previous studies. Bilateral independent IED are associated with worse surgical outcome after temporal lobectomy compared with unitemporal spike foci. ${ }^{24} 25$ The reason for this could be that they represent an extended epileptogenic region or a greater epileptogenicity, as they are more often associated not only with a worse surgical outcome but also with bilateral seizure onset zone, bilateral seizure propagation, and greater seizure frequency. ${ }^{30}{ }^{31}$ Some studies have found that patients with generalised epilepsies (which are typically associated with generalised IED) may obtain some benefit from VNS treatment, although seizure freedom was infrequently reported..$^{911} 123233$ On the other hand, Labar found that Lennox-Gastaut syndrome might be associated with unresponsiveness to VNS treatment. ${ }^{10}$

Although only four of our patients had symptomatic generalised epilepsy, generalised IED occurred in 14 other patients with focal epilepsy. Generalised (bilateral synchronous) IED in focal epilepsy is termed secondary bilateral synchrony. ${ }^{34}$ Some studies found them related to worse surgical outcome in frontal ${ }^{26}$ and temporal ${ }^{35}{ }^{36}$ epilepsy. The pathomechanism of bilateral synchronous IED in focal epilepsy is not clear, but it is likely that discharges from the primary focus spread through the thalamus or through the corpus callosum to the corresponding contralateral area, producing a nearly synchronised discharge. ${ }^{37}{ }^{38}$ An alternative explanation is that bilateral synchronous IED arise from an interaction of multiple active foci. ${ }^{39}$ Our findings of a correlation between lack of bilateral (independent and dependent) IED and favourable VNS outcome support the assumption that bilateral IED represent a higher degree of epileptogenicity. 
Two large multicentre studies showed that patients with a long duration of epilepsy had less chance of being seizurefree on VNS treatment. ${ }^{75}$ On the other hand, another study found that VNS responsiveness was associated with a longer duration of epilepsy..$^{10}$ Although the epilepsy duration in our study showed no significant influence on the outcome, we found a non-significant trend towards a negative association between longer illness duration and VNS outcome.

One may speculate that the intrinsic epileptogenicity of MCD-which is accompanied by altered functional properties $^{40}{ }^{41}$ different from other epileptogenic lesions-might be associated with altered pharmacology or response to VNS. In the single predictive variable analyses, we found that the presence of MCD was associated with favourable VNS outcome; however, the logistic regression analysis did not confirm this result.

A relatively large number of our patients became seizurefree compared with other studies in this field. One explanation for this may be that the outcome results of long term and open VNS studies have tended to be better than in controlled and short term investigations. ${ }^{3-13}$ Another possibility is that there was a large number of patients with lesional epilepsy in our study compared with other studies. This might explain the better outcome. Indeed, abnormal MRI predicts a better outcome in epilepsy surgery. ${ }^{24}{ }^{26}$ Conversely, the epilepsies with most frequent aetiologies found in our study, such as MCD and hippocampal sclerosis, are poorly controlled by antiepileptic drugs compared with epilepsies with normal MRI findings. ${ }^{42}$

In two patients, seizure freedom was achieved after introducing levetiracetam. In both patients the VNS initially resulted in a benefit (the first patient had $>50 \%$ seizure reduction, the second a two month initial seizure freedom), but final seizure freedom was only achieved directly after the introduction of levetiracetam. The possible positive effect of the newly introduced drug levetiracetam on seizure-free outcome was not significant owing to the limited statistical power of this study, which precluded adequate analysis of the effect of new antiepileptic drugs. In another reported patient receiving VNS treatment, seizure freedom occurred after adding lamotrigin. ${ }^{5}$ Further studies are required to determine whether there are some specific VNS antiepileptic drug synergistic effects.

Our results may have been influenced by the fact that there were no restrictions on changes in concomitant antiepileptic drugs, and adjustments of concomitant drugs were at the discretion of the treating physician. This is one of the limitations of our study. Conversely, in long term outcome studies it is impossible to stabilise the antiepileptic drug regime. This is true for long term studies investigating surgical $^{4344}$ or VNS ${ }^{513}$ outcomes. Fixing the antiepileptic regimen would not only be a clinical but also a theoretical failure because it would have resulted in excluding patients who needed a change in their antiepileptic drug treatment. The effectiveness of VNS, however, improves over time. ${ }^{3}$ Thus some patients with long term improvement would have been excluded. The way to address this issue is to compare a group of patients receiving medical treatment only with a group of VNS patients, as was done in the study that proved the effectiveness of temporal lobectomy. ${ }^{45}$ The retrospective nature of our study, however, did not make it possible to include such a control group.

Our results are preliminary because they were based on a relatively small patient population. However, with respect to other single centre VNS studies, only one included more patients with long term outcome data. ${ }^{12}$ The relatively small number of patients resulted in limited statistical power. Thus some variables such as bilateral independent seizure pattern (table 3), tuberous sclerosis, perinatal stroke, or hypothalamic hamartoma (table 4) did not occur with a great enough frequency to reach statistical significance, although all of these occurred only in non-seizure-free patients. Our study may facilitate other prospective investigations enrolling larger numbers of patients.

\section{ACKNOWLEDGEMENTS}

This work was supported by a grant from the Deutsche Forschungsgemeinschaft (DFG-Eb 111/2-2)

\section{Authors' affiliations}

J Janszky, M Hoppe, F Behne, I Tuxhorn, H W Pannek, A Ebner, Bethel Epilepsy Centre, Bielefeld, Germany

Competing interests: none declared

\section{REFERENCES}

1 Handfort A, DeGiorgio CM, Schachter SC, et al. Vagus nerve stimulation therapy for partial-onset seizures. A randomized active-control trial. Neurology 1998;51:48-55.

2 Ben-Menachem E, Manon-Espaillat R, Ristanovic R, et al. Vagus nerve stimulation for treatment of partial seizures. 1. A controlled study of effect on seizures. Epilepsia 1994;35:616-26.

3 Boon P, Vonck K, Reuck J, et al. Vagus nerve stimulation for refractory epilepsy. Seizure 2001;10:448-55.

4 Murphy JV, Torkelson R, Dowler I, et al. Vagal nerve stimulation in refractory epilepsy. The first 100 patients receiving vagal nerve stimulation at a pediatric epilepsy center. Arch Pediatr Adolesc Med 2003;157:560-4.

5 Vonck $K$, Boon $P, D^{\prime}$ Havé $M$, et al. Long-term results of vagus nerve stimulation in refractory epilepsy. Seizure 1999;8:328-34.

6 Uthman BM, Wilder BJ, Penry JK, et al. Treatment of epilepsy by stimulation of the vagal nerve. Neurology 1993;43:1338-45.

7 Helmers SL, Griesemer DA, Dean JC, et al. Observations on the use of vagus nerve stimulation earlier in the course of pharmacoresistant epilepsy: patients with seizure for six years or less. Neurologist 2003;9:160-4.

8 Lundgren J, Amark P, Blennow G, et al. Vagus nerve stimulation in 16 children with refractory epilepsy. Epilepsia 1998;39:809-13.

9 Labar D, Murphy J, Tecoma E, E VNS Study group. Vagus nerve stimulation for medication-resistant generalized epilepsy. Neurology 1999;52:1510-15.

10 Labar D. Vagus nerve stimulation for 1 year in 269 patients on unchanged antiepileptic drugs. Seizure 2004; 13:392-8.

11 Frost M, Gates J, Helmers SL. Vagus nerve stimulation in children with refractory seizures associated with Lennox-Gastaut syndrome. Epilepsia 2001;42:1148-52.

12 Scherrmann J, Hoppe C, Kral T, et al. Vagus nerve stimulation. Clinical experience in a large patient series. J Clin Neurophysiol 2001;18:408-14.

13 Nakken KO, Henriksen O, Roste GK, et al. Vagal nerve stimulation - the Norwegian experience. Seizure 2003;12:37-41.

14 Schachter SC. Vagus nerve stimulation therapy summary - five years after FDA approval. Neurology 2002;59(suppl 4):S15-20.

15 Ranfroe JB, Wheless JW. Earlier use of adjunctive vagus nerve stimulation for refractory epilepsy. Neurology 2002;59(suppl 4):S26-30.

16 DeGiorgio CM, Schachter SC, Handforth A, et al. Prospective long-term study of vagus nerve stimulation for the treatment of refractory seizures. Epilepsia 2000;41:1195-2000.

17 George R, Salinsky M, Kuzniecky R, et al. Vagus nerve stimulation for treatment of partial seizures. 3. Long-term follow-up on first 67 patients exiting a controlled study. Epilepsia 1994;35:637-43.

18 Ben-Menachem M, Hellström K, Waldton C, et al. Evaluation of refractory epilepsy treated with vagus nerve stimulation for up to 5 years. Neurology 1999;52:1265-7.

19 Birbeck GL, Hays RD, Cui X, et al. Seizure reduction and quality of life improvements in people with epilepsy. Epilepsia 2002;43:535-8.

20 Leidy NK, Elixhauser A, Vickerey B, et al. Seizure frequency and the healthrelated quality of life of adults with epilepsy. Neurology 1999;53:162-6.

21 Attarian H, Dowling J, Carter J, et al. Video EEG monitoring prior to vagal nerve stimulation implantation. Neurology 2003;61:402-3.

22 Landy HJ, Ramsay RE, Slater J, et al. Vagus nerve stimulation for complex partial seizures: surgical technique, safety and efficacy. J Neurosurg 1993;78:26-31.

23 American electroencephalographic society. Guideline for standard electrode position nomenclature. J Clin Neurophysiol 1994;11:111-13.

24 Radhakrishnan K, So EL, Silbert PL, et al. Predictors of outcome of anterior temporal lobectomy for intractable epilepsy - a multivariate study. Neurology 1998;51:465-71

25 Schulz R, Lüders $\mathrm{HO}$, Hoppe $M$, et al. Interictal EEG and ictal scalp EEG propagation are highly predictive of surgical outcome in mesial temporal lobe epilepsy. Epilepsia 2000;41:564-70.

26 Janszky J, Jokeit $\mathrm{H}$, Schulz R, et al. EEG predicts surgical outcome in lesional frontal lobe epilepsy. Neurology 2000;54:1470-6.

27 Degen $R$, Ebner $A$, Lahl R, et al. MRI and EEG findings in surgically treated patients with partial seizures due to neuronal migration disorders, their relations to each other and to surgery outcome. Acta Neurol Scand 2003; 108:309-18. 
28 Alsaadi TM, Laxer KD, Barbaro NM, et al. Vagus nerve stimulation for the treatment of bilateral independent temporal lobe epilepsy. Epilepsia $2001 ; 42: 954-6$. 29 Kuba R, Brazdil M, Novak Z, et al. Effect of vagal nerve stimulation on patients with bitemporal epilepsy. Eur J Neurol 2003;10:91-4.

30 Janszky J, Fogarasi $A$, Jokeit $H$, et al. Spatiotemporal relationship between seizure activity and interictal spikes in temporal lobe epilepsy. Epilesy Res 2001;47:179-88.

31 Janszky J, Rásonyi G, Clemens Z, et al. Clinical differences in patients with hippocampal sclerosis and unitemporal or bitemporal epileptiform discharges. Seizure 2003;12:550-4.

32 Labar D, Nikolov B, Tarver B, et al. Vagus nerve stimulation for symptomatic generalized epilepsy: a pilot study. Epilepsia 1998;39:201-5.

33 Wheless JW, Maggio V. Vagus nerve stimulation therapy in patients younger than 18 years. Neurology 2002:59(suppl 4):S21-25.

34 Tükel K, Jasper H. The EEG in parasagital lesions. Electroencephalogr Clin Neurophysiol 1952;4:481-94.

35 Guldvog B, Løyning Y, Hauglie-Hanssen E, et al. Predictive factors for success in surgical treatment for partial epilepsy: a multivariate analysis. Epilepsia 1994:35:566-78.

36 Lieb JP, Engel J, Gevins A, et al. Surface and deep EEG correlates of surgical outcome in temporal lobe epilepsy. Epilepsia 1981;22:515-38.

37 Spencer SS, Spencer DD, Williamson PD, et al. Effects of corpus callosum section on secondary bilaterally synchronous interictal EEG discharges. Neurology 1985;35:1689-94.
38 Kobayashi K, Ohtsuka Y, Oka E, et al. Primary and secondary bilateral synchrony in epilepsy: differentiation by estimation of interhemispheric small time differences during short spike-wave activity. Electroencephalogr Clin Neurophysiol 1992;83:93-103.

39 Blume WT, Pillay N. Electrographic and clinical correlates of secondary bilateral synchrony. Epilepsia 1985;26:636-41.

40 Palmini A, Gambrardella A, Andermann F. Intrinsic epileptogenicity of human dysplastic cortex as suggested by corticography and surgical results. Ann Neurol 1995; 37:476-87.

41 Janszky J, Ebner A, Kruse B, et al. The functional organization of the brain with malformations of cortical development Ann Neurol 2003;53:759-67

42 Semah F, Picot MC, Adam C, et al. Is the underlying cause of epilepsy a major prognostic factor for recurrence? Neurology 1998;51:1256-62.

43 Salanova V, Andermann F, Rasmussen T, et al. The running down phenomenon in temporal lobe epilepsy. Brain 1996;1 19:989-96.

44 Hennessy MJ, Elwes RD, Honavar M, et al. Predictors of outcome and pathological considerations in the surgical treatment of intractable epilepsy associated with temporal lobe lesions. J Neurol Neurosurg Psychiatry 2001;70:450-8.

45 Wiebe S, Blume WT, Girvin JP, et al for the Effectiveness and Efficiency of Surgery for Temporal Lobe Epilepsy Study Group. A randomized, controlled trial of surgery for temporal-lobe epilepsy. N Engl J Med $2001 ; 345: 311-18$.

\section{HISTORICAL NOTE}

\section{Neuralgic amyotrophy}

S ir Charles Bell first drew attention to the long thoracic nerve in $1821 .{ }^{1}$ Lesions of the nerve cause winging of the scapula with medial transition of the scapula and prominence of the vertebral border. ${ }^{2}$ Skillern in 1913 first observed that the nerve is exposed to trauma in the root of the neck. He wrote:

"The long thoracic nerve is exposed to trauma as it traverses the scalenius medius."

The nerve itself is small in diameter and fragile and is vulnerable at many sites, particularly within the scalenus medius. ${ }^{3}$

The commonest long thoracic nerve lesion encountered by neurologists is acute brachial plexus neuropathy (neuralgic amyotrophy), which is less common than trauma, accounting for only $5-10 \%$ of all cases. ${ }^{4}$ In 1943, describing 46 patients with "localised neuritis of the shoulder girdle" Spillane ${ }^{5}$ gave the first full description, elegantly summarised in his renowned text: ${ }^{6}$

"There is acute onset of pain in the shoulder and side of the neck, over the scapula and down the affected arm usually not further than the elbow... The pain is commonly worse at night, disturbing or preventing sleep... and usually persists for a week or 10 days... little or no general upset, no stiffness of the neck and upper limb reflexes may remain unaltered. After a few days or when the pain is subsiding, paralysis supervenes. The muscles most commonly affected are the serratus, the spinati, the deltoid and the trapezii in that order. The neuritis may be bilateral...Sensory loss is as a rule slight or absent; a small zone of hypo-aesthesia over an affected deltoid muscle... The prognosis is good but affected muscles often waste rapidly and recovery is slow...a year or more. Rarely there may be a recurrence in the same or opposite shoulder."

A year later Aldren Turner ${ }^{7}$ provided a similar account. The disorder is sometimes named after Parsonage and Turner, ${ }^{8}$ doi: $10.1136 /$ jnnp. 2004.042531

who provided further examples. In 66 of their original 136 patients, they found possible precipitating causes that included operations, infections, inoculations, and trauma to remote areas. Familial, post-infective (for example influenza, coxsackievirus, infectious mononucleosis, Borrelia burgdorferi) and post-vaccination factors have been described, but the aetiology is unknown in many examples. Meuleman et al suggested that Dreschfeld ${ }^{9}$ (1885) may have published the first report of hereditary neuralgic amyotrophy, that of a 43 year old woman who had suffered three episodes of painful upper limb weakness and whose sister had suffered seven similar attacks.

A possible earlier example was described as "spontaneous parenchymatous neuritis" in which Joffroy in 1879 showed loss of myelinated fibres. ${ }^{10}$

304 Beverley Road, Analby, Hull HU10 7BG, UK; jmspearce@freenet.co.uk

\section{References}

1 Bell C. Of the nerves, giving an account of some experiments on their structure and functions, which lead to new arrangement of the system. Philosophical Transactions of the Royal Society of London 1821;111:398-424. Cited in: Norman JM, ed. Garrison \& Morton's Medical Bibliography. 5th edn. Aldershot: Scolar Press, 1991;1255:200.

2 Wiater JM, Flatow EL. Long thoracic nerve injury. Clin Orthop 1999;368:17-27.

3 Birch R, Bonney G, Wynn Parry CB. Entrapment neuropathy. In: Surgical disorders of the peripheral nerves. New York: Churchill Livingstone, 1998:245-92.

4 Skillern PG. Serratus magnus palsy with proposal of a new operation for intractable cases. Ann Surg 1913;57:909-15.

5 Spillane JD. Localised neuritis of the shoulder girdle. Lancet 1943;ii:532-5.

6 Spillane JD. An atlas of clinical neurology. Oxford: Oxford University Press, 1968:134-5.

7 Turner JWA. Acute brachial radiculitis. BMJ 1944;2:592-4.

8 Parsonage MJ, Turner JWA. Neuralgic amyotrophy: the shoulder-girdle syndrome. Lancet 1948;1:973-8.

9 Dreschfeld J. On some rarer forms of muscular atrophies. Brain 1885;8:164-90.

10 Joffroy A. De la névrite parenchymateuse spontanée, géneralisée ou partielle. Archives Physiol, Norm Path 1879;6:172-98. 